Reprod. Nutr. Dévelop. 1980, 20 (4 A), 961-973.

\title{
Le fonctionnement du système mitochondrial et la respiration au 5e stade larvaire chez Pieris Brassicae (Lépidoptère)
}

\author{
par C. GUILLET, J. FOURCHE \\ avec la collaboration technique de D. GUILLET
}

\begin{abstract}
Département de Biologie générale et appliquée, Laboratoire associé au C.N.R.S. $n^{\circ} 243$, Université Claude Bernard Lyon-I 43, Boulevard du 11 novembre 1918, 69622 Villeurbanne Cedex, France.
\end{abstract}

Summary. Functioning of the mitochondrial system and respiration at the fifth instar in Pieris brassicae (Lepidoptera).

The profiles of respiratory rate, total proteins, mitochondrial proteins and mitochondrial activity have been described during the fifth instar of Pieris brassicae reared either under long-day or short-day photoperiod. There was no fundamental difference per unit of live weight between the long-day and short-day larvae.

The biochemical characteristics of the larval mitochondria have been described. and the mitochondria were shown to oxidize succinate better than $\alpha$-glycerophosphate.

Two periods during the fifth instar were related to mitochondrial activity. During the first one, this specific activity was high during 50 to 60 p. 100 of the instar ; it then decreased. This alteration was superimposed on changes in the hormonal balance.

It is shown that the respiratory rate was not only a passive response to the energy demand, and that specific mitochondrial activity must be considered as a parameter of the development program.

\section{Introduction.}

Chez les organismes aérobies, la respiration fournit l'essentiel de l'énergie. Elle couvre les différents besoins de l'organisme résultant du métabolisme de maintien, des différentes fonctions organiques et des diverses activités cellulaires (Harvey, 1962). Chez les insectes, l'évolution de la respiration, qui constitue une mesure globale du métabolisme, est caractéristique de chacune des étapes du développement : embryogenèse (Fourche, 1969), croissance organique (Fourche ef Ambrosioni, 1969) ou organismique (Fourche, 1965), métamorphose (Fourche, 1967a). La respiration est également tributaire de l'état physiologique, comme l'inanition chez la larve (Bosquet, 1971), la reproduction (Slama, 1968), le vol chez les imagos (Wigglesworth, 1949), la diapause (Schneiderman et Williams, 1953 ; Guillet, 1976). Les variations de la respiration traduisent finalement l'ajustement à chaque instant de la production d'énergie aux besoins de l'organisme. 
La nature de la respiration, échange de l'organisme avec le milieu extérieur, peut rapidement conduire à l'idée que son intensité est seulement la résultante de l'ensemble des phénomènes métaboliques qui interviennent en amont dans l'organisme. Autrement dit, la respiration suivrait de manière strictement passive la demande énergétique (Harvey, 1962). Dans ces conditions, contrairement aux idées de Fourche (1969) qui suggérait chez les nymphes des mécanismes propres de réglage intégré, Steele (1976) conclut que la respiration échappe au contrôle programmé du développement.

Les mitochondries constituent certainement le site essentiel de la production d'énergie. Une mitochondriogenèse active est décrite lors de la mue dans divers organes (Wigglesworth, 1959 ; Larsen, 1970) ou lors des métamorphoses en particulier dans la musculature alaire (Walker et Birt, 1969 ; Herold, 1965 ; Kunkel, 1975). La mitochondriogenèse est nécessaire à la morphogenèse normale. En effet, toule perturbation de l'augmentation des mitochondries (Holmes ef Keeley, 1975) altère le développement, l'arrête ou entraîne une mort précoce de l'animal (Keeley et Olson, 1977). Au cours de la vie larvaire de la Drosophile, Rezvoy (1977) a observé une augmentation parallèle des quantités de protéines mitochondriales et de cytochrome $C$; mais la Drosophile constitue certainement un cas particulier dans la mesure où la croissance de la larve se fait essentiellement par augmentation de la taille des cellules. Ces différents résultats conduisent à l'idée que les capacités respiratoires de l'insecte, liées aux mitochondries, sont un des éléments programmés du déve loppement.

Nous avons choisi d'aborder cette question au cours du dernier âge larvaire de Pieris brassicae, chez qui la croissance est différente suivant que les animaux doivent ou non entrer en diapause nymphale, en fonction de la photopériode appliquée aux chenilles. L'expérimentation comprend l'étude de la respiration des larves intactes, de la respiration in vitro d'homogénat des larves, de la respiration in vitro des mitochondries extraites par centrifugation différentielle. Les trois mesures donnent des évolutions de même type. Ensuite, nous montrons que la quantité des protéines mitochondriales augmente et que cette augmentation s'intègre parfaitement dans la croissance estimée par la mesure du poids vif ou des protéines totales. Enfin, certaines activités mitochondriales sont étudiées qui permettent de définir deux phases qui se superposent, chez la larve, à deux périodes de signification physiologique distincte. Ces différents résultats nous conduisent à l'idée que la respiration n'échappe pas au programme de développement.

\section{Matériel et méthodes.}

Animaux. - Les larves sont synchronisées à la 4e exuviation larvaire. Elles sont nourries « ad libitum » de feuilles de choux et sont élevées à $20^{\circ} \mathrm{C}$ (Guillet, 1976), soit en photopériode longue, $16 \mathrm{~h}$ d'éclairement, $8 \mathrm{~h}$ d'obscurité (larves $\mathrm{PL}$ ), soit en photopériode courte, $8 \mathrm{~h}$ d'éclairement, $16 \mathrm{~h}$ d'obscurité (larves PC). Dans le premier cas, la mue larvo-nymphale intervient 5 jours après la $4^{\mathrm{e}}$ exuviation larvaire ef les animaux ont un développement continu. Dans le second cas, la durée du 5 e stade est de 7,5 jours ef les nymphes entrent en diapause. 
Dans les expériences de respirométrie et de mesure d'activité mitochondriale, les larves sont prélevées $3 \mathrm{~h}$ après le début de l'éclairement. Pour le dosage des protéines, la régularité des prélèvements n'a pas été aussi sévère de façon à intercaler les mesures faites sur les deux groupes d'animaux.

Méthodes. - La procédure utilisée est résumée dans le tableau 1.

TABLEAU 1

Procédure expérimentole

$8 \mathrm{~h}$

$16 \mathrm{~h}$

Eclairement

7

Eclairement

Larves d'âge connú à partir

de la $4^{\mathrm{C}}$ exuviation larvaire

Respiromètre $25^{\circ} \mathrm{C}$

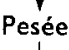

$\downarrow$

Dissection

Elimination du contenu du tube digestif

$\downarrow \longrightarrow$ Broyage dans ATC 10 p. 100

Broyage dans milieu d'extraction $0-4^{\circ} \mathrm{C}$ $\downarrow$

Centrifugation $0-4^{\circ} \mathrm{C}$

Filtration

Centrifugation

$10000 \mathrm{~g}, 15 \mathrm{~min}$

$\downarrow 600 \mathrm{~g}, 15 \mathrm{~min}$
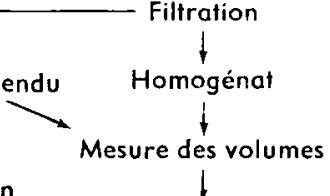

Culot resuspendu Surnageant éliminé Oxygraphe $25^{\circ} \mathrm{C}$ $(10 \mathrm{ml})$

Centrifugation $0-4^{\circ} \mathrm{C}$

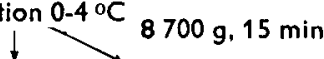

Respiration succinate avec ou sans ADP

Culot resuspendu Surnageant éliminé 500-800 $\mu 1$

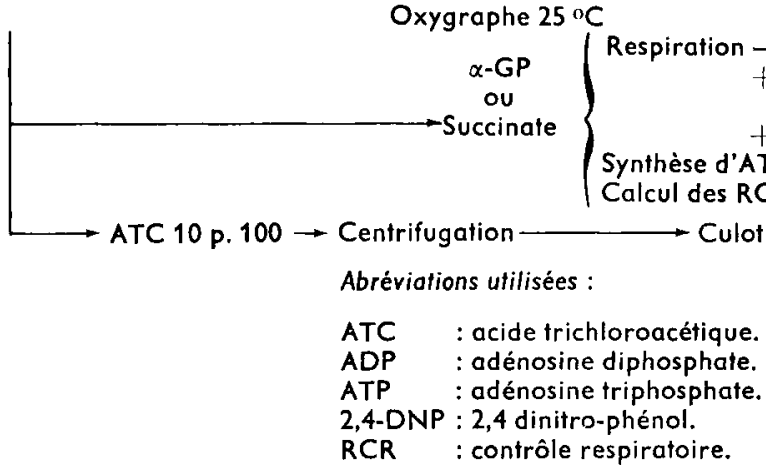

Culot $\mathrm{Na}$ OH $2 \mathrm{~N}, 100^{\circ} \mathrm{C}$

$2 \times 1 \mathrm{~h}$

1

Dosage des protéines

RCR : contrôle respiratoire.

- Les mesures de respiration sont effectuées à $25^{\circ} \mathrm{C}$ à l'aide d'un respiromètre automatique (Fourche ef al., 1972). La valeur retenue se situe 3 h 30 après la mise en place des larves : entre 1 et 5 dans chaque cellule.

- Le milieu d'extraction des mitochondries à pH 7,4 est composé de saccharose $250 \mathrm{mM}$, EDTA $1 \mathrm{mM}$, Tris $50 \mathrm{mM}$. 
- La respiration des différentes suspensions est mesurée polarographiquement à $25^{\circ} \mathrm{C}$, à l'aide d'un oxygraphe Gilson muni d'une électrode de Clark, dans une cellule de $1,6 \mathrm{ml}$.

Le milieu réactionnel à $\mathrm{pH} 7,4$ contient : saccharose $250 \mathrm{mM}$, EDTA $1 \mathrm{mM}$, Tris $50 \mathrm{mM}, \mathrm{MgCl}_{2} 8 \mathrm{mM}, \mathrm{KCl} 80 \mathrm{mM}$, albumine bovine $0,5 \mathrm{p} .100$. A $1 \mathrm{ml}$ de ce milieu, on ajoute dans la cellule $100 \mu$ l de phosphate de potassium à $\mathrm{pH} 7,4$ ef $50 \mu l$ de substrat $1 \mathrm{M}$ à pH 7,4 (succinate ou $\alpha$-glycérophosphate sous forme de sel disodique). La réaction débute avec l'addition de 50 à $100 \mu l$ de la suspension. Trois minutes plus tard, on ajoute $20 \mu \mathrm{l}$ d'ADP $6 \mathrm{mM}$. La respiration des mitochondries est mesurée sur les enregistrements oxygraphiques, avant addition d'ADP, après addition d'ADP (état 3) et à l'état 4 de retour. Le contrôle respiratoire est obtenu en faisant le rapport des respirations état 3/état 4 (Estabrook, 1967).

Le 2,4-DNP est utilisé à la concentration finale de $10^{-4} \mathrm{M}$ et l'oligomycine à raison de 1 à $2 \mu \mathrm{g}$ par $\mathrm{mg}$ de protéines mitochondriales.

- La synthèse d'ATP est calculée connaissant la quantité d'ADP fournie ef l'intervalle de temps qui sépare le début de l'état 3 du retour à l'état 4 mesuré sur les enregistrements oxygraphiques. Elle est exprimée en nano-moles par minute et par mg de protéines mitochondriales.

Dans ces expériences 10 à 25 larves sont nécessaires pour chaque point.

- Les protéines tolales sont extraites à partir d'animaux isolés.

- Les protéines sont dosées selon Lowry et al. (1951).

\section{Résultats.}

1. Comparaison des respirations de la larve intacte, de l'homogénat et de la fraction mitochondriale (fig. 1).

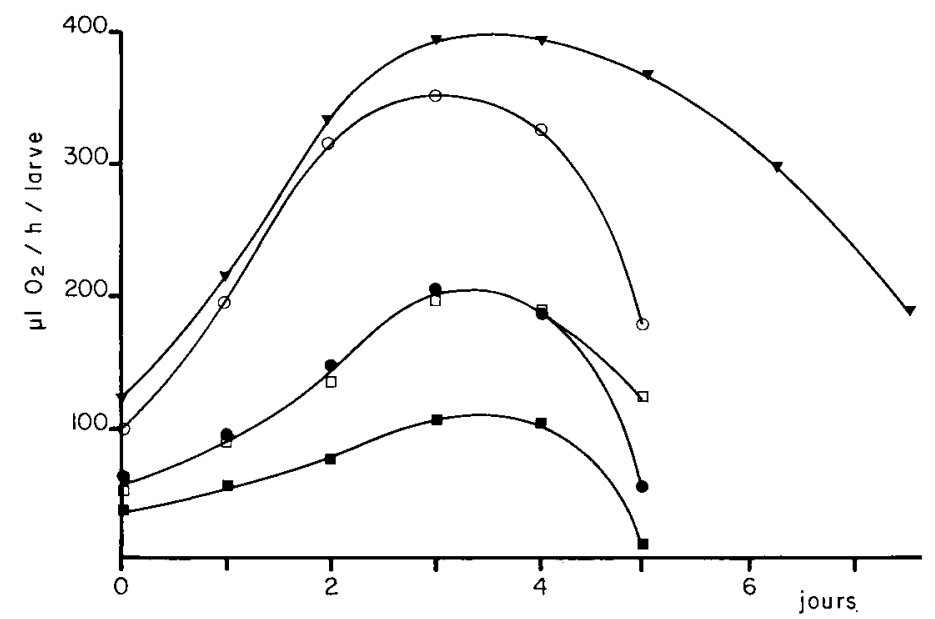

FIG. 1. - Evolution de l'intensité respiratoire de la larve intacte $P L \circ, P C \nabla$. Evolution chez la larve $P L$, des respirations de l'homogénat $\mathrm{a}$, du culot $8700 \mathrm{~g}$ a, de la somme des respirations des culots $600 \mathrm{~g}+8700 \mathrm{~g}$ - Ces mesures sont effectuées en présence de succinate et d'ADP. 
Chez les larves $\mathrm{PL}$, la respiration est de $108 \mu \mathrm{l} \cdot \mathrm{h}^{-1}$ au début du $5^{\mathrm{e}}$ stade. Elle afteint son maximum au jour $3: 350 \mu \mathrm{l} . \mathrm{h}^{-1}$, puis elle décroît jusqu'à $180 \mu \mathrm{l} . \mathrm{h}^{-1}$ chez la prénymphe.

Chez les larves PC, l'évolution est du même type : $115 \mu \mathrm{l} . \mathrm{h}^{-1}$ après la dernière exuviation larvaire, $395 \mu \mathrm{l} . \mathrm{h}^{-1}$ au jour 4 ef $192 \mu \mathrm{l} . \mathrm{h}^{-1}$ chez la prénymphe.

Chez les larves PL, la respiration de l'homogénat qui est calculée connaissant la respiration d'une partie aliquote et le volume total de l'homogénat, passe de $50 \mu \mathrm{l} . \mathrm{h}^{-1}$ à $200 \mu \mathrm{l} . \mathrm{h}^{-1}$ au jour 3 et $125 \mu \mathrm{l} \cdot \mathrm{h}^{-1}$ chez la prénymphe.

Si l'homogénat subit le cycle complet de centrifugation $600 \mathrm{~g}+2$ fois $8700 \mathrm{~g}$, la respiration mesurée, après remise en suspension, est comparable à celle obtenue avec l'homogénat non centrifugé sauf pour le point prénymphe pour lequel l'activité diminue de 50 p. 100 . La respiration de la suspension mitochondriale préparée à partir du culol $8700 \mathrm{~g}$ passe aussi par un maximum au jour 3 .

II existe une relation linéaire entre la respiration de la larve $(R L)$ ef celle de l'homogénat $(\mathrm{RH})$ pendant la totalité de l'âge $R L=1,61 \mathrm{RH}+32,85, r=0,91$.

La respiration de la suspension mitochondriale $(R M)$ est linéairement liée à la respiration de l'homogénat sauf pour les prénymphes, $\mathrm{RM}=0,512 \mathrm{RH}+7,304$, $r=0,999$.

2. Estimation de quelques paramètres de la croissance.

- Evolution du poids vif (fig. 2). Le poids vif des larves PC, est significativement plus élevé que celui des larves PL en fin de croissance. Le poids maximum est atteint

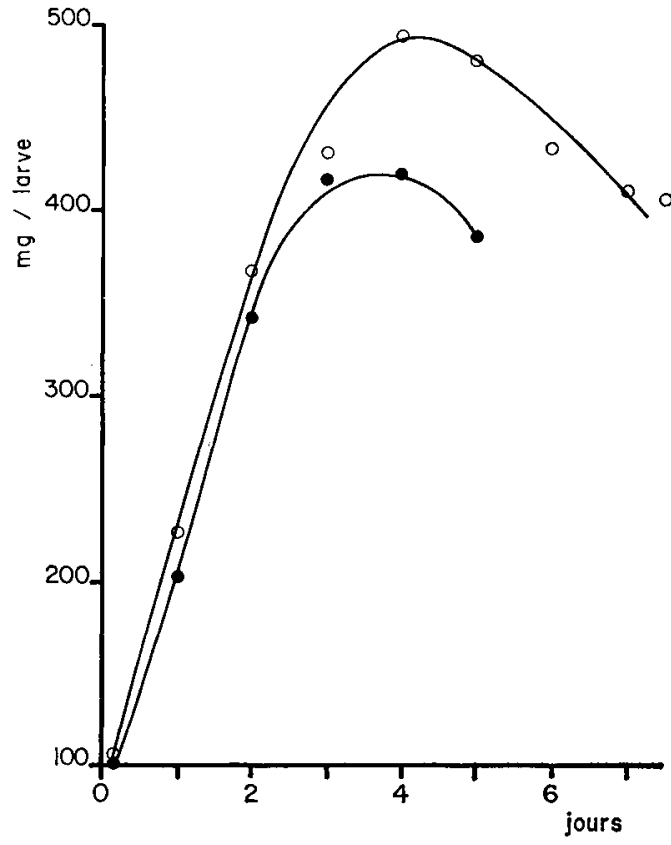

FIG. 2. - Evolution du poids vif moyen d'une larve ou 5e stade. Larve PL •, Larve PCo. Ces valeurs sont obtenues à partir des différents lots de larves utilisées pour extraire les mitochondries. 
aux jours 3 ef 4 respectivement chez les larves PL ef PC, soit à environ 60 p. 100 de la durée du 5 e stade. La prise de nourriture se poursuit un jour de plus sans que le poids vif augmente.

- Evolution des protéines totales (fig. 3). Elles évoluent selon une sigmoïde classique : de $6 \mathrm{mg}$ par larve à la 4 e exuviation larvaire à $32-34 \mathrm{mg}$ à la fin du 5 e stade.

- Evolution des protéines mitochondriales du culot $8700 \mathrm{~g}$ (fig. 4). Cette méthode a été employée pour quantifier les mitochondries extraites par centrifugation différentielle (Holmes et Keeley, 1975). Chez les larves PL, la quantité de protéines mitochondriales passe de $215 \mu \mathrm{g}$ par larve au début du 5e stade à $707 \mu \mathrm{g}$ chez les larves de 3 jours ; puis elle décroît jusqu'à la mue larvo-nymphale où elle atteint $600 \mu \mathrm{g}$. Chez les larves PC, on passe de $265 \mu \mathrm{g}$ à $875 \mu \mathrm{g} 4$ jours après la $4 \mathrm{e}$ exuviation larvaire et $650 \mu \mathrm{g}$ peu avant l'exuviation larvo-nymphale.

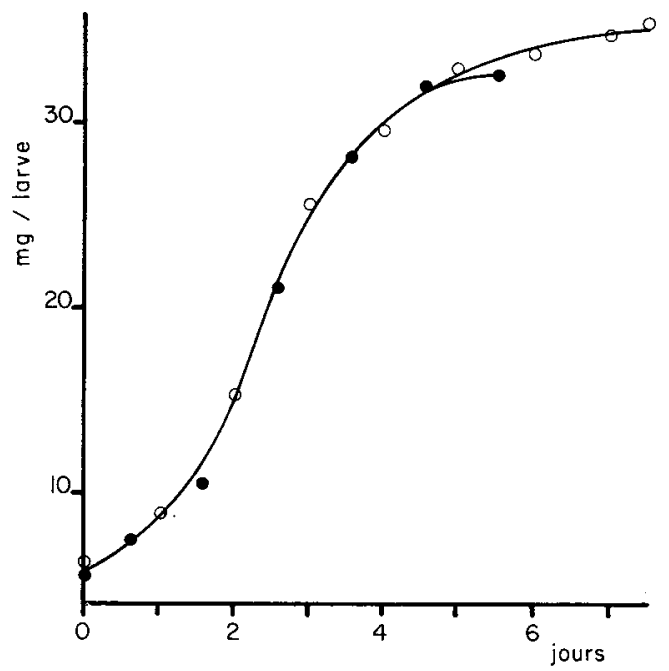

FIG. 3.

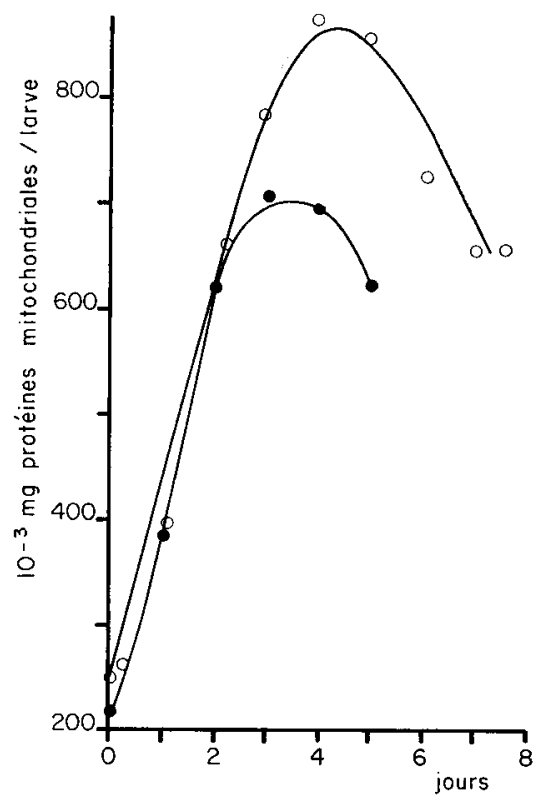

FIG. 4.

FIG. 3. - Evolution des protéines totales d'une larve au 5e stade. Larve PL •, Larve PC o. Les protéines sont extraites à partir d'individus isolés.

FIG. 4. - Evolution des protéines mitochondriales du culot $8700 \mathrm{~g}$. Larve PL •, Larve PCo.

- Relation protéines totales-protéines mitochondriales. Si l'on exprime les protéines totales et les protéines mitochondriales en valeur relative (100 après la 4 ex exuviation larvaire) et que l'on transforme ces données en valeurs logarithmiques, on montre que ces valeurs se répartissent autour de la même droite pendant toute la période d'augmentation du poids vif des larves. 


\section{Caractéristiques des mitochondries larvaires.}

Plusieurs substrats ont été testés. Avec le pyruvate, l'oxoglutarate ou le citrate, la respiration est très faible. L' $\alpha$-glycérophosphate ef le succinate qui se sont révélés plus efficaces ont été retenus.

D'une manière générale, la respiration des mitochondries est stimulée par l'ADP et le 2,4-dinitrophénol, la synthèse d'ATP est inhibée par l'oligomycine, seules les mitochondries de prénymphes ne réagissent pas à l'addition d'ADP ou de 2,4-DNP. Ces mitochondries apparaissent alors découplées.

3a) Respiration spécifique des mitochondries.

Larves PL (fig. 5). En présence d' $\alpha$-glycérophosphate (fig. $5 \mathrm{~A}$ ), la respiration oscille autour de $32 \mu \mathrm{l} . \mathrm{h}^{-1} . \mathrm{mg}^{-1}$ de protéines, pendant 3 jours, avant addition d'ADP, puis décroît jusqu'à $12 \mu \mathrm{l} \cdot \mathrm{h}^{-1} \cdot \mathrm{mg}^{-1}$ avant la mue larvo-nymphale. A l'état 3 (en présence d'ADP), la respiration décroît lentement pendant 4 jours.

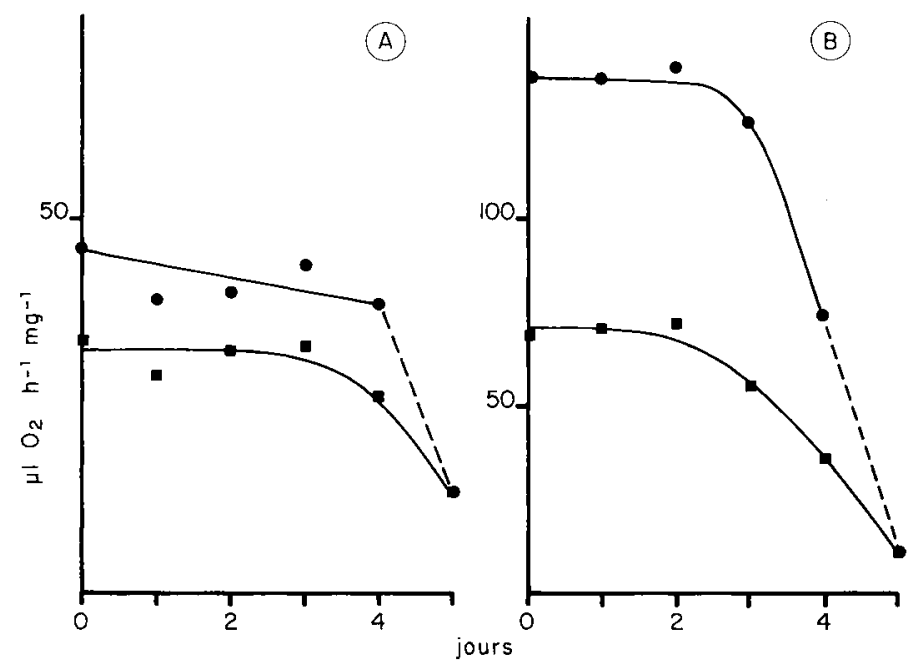

FIG. 5. - Activité respiratoire spécifique des mitochondries des larves $P L$ au cours du $5^{e}$ âge ovant च ef après • l'addition d'ADP. (A) substrat $=\alpha$-glycérophosphate, (B) substraf = succinate.

En présence de succinate (fig. 5B), la respiration des mitochondries est nettement plus élevée qu'en présence d' $\alpha$-glycérophosphate. Avant addition d'ADP, elle est de $70 \mu \mathrm{l} . \mathrm{h}^{-1} . \mathrm{mg}^{-1}$ jusqu'au $3^{\mathrm{e}}$ jour, puis elle diminue jusqu'à $10 \mu \mathrm{l} . \mathrm{h}^{-1} \cdot \mathrm{mg}^{-1}$ au $5^{\mathrm{e}}$ jour. Les variations se font dans le même sens lorsque la respiration est mesurée à l'état 3 (de 140 à $75 \mu \mathrm{l} . \mathrm{h}^{-1} \cdot \mathrm{mg}^{-1}$ ).

Larves PC. En présence de succinate (fig. 6), la respiration est de $70 \mu \mathrm{l} \cdot \mathrm{h}^{-1} \cdot \mathrm{mg}^{-1}$ avant addition d'ADP. Cette valeur ne change pas avant le $4^{e}$ jour. Elle décroît jusqu'à $13 \mu \mathrm{l} . \mathrm{h}^{-1} \cdot \mathrm{mg}^{-1}$ chez la prénymphe. A l'étał 3 , la respiration est de $145 \mu \mathrm{l} . \mathrm{h}^{-1} \cdot \mathrm{mg}^{-1}$ pendant 3 jours. Elle n'est plus que de $100 \mu \mathrm{l} . \mathrm{h}^{-1} \cdot \mathrm{mg}^{-1} 6$ jours après la mue larvaire. 
En présence d' $\alpha$-glycérophosphate, les valeurs initiale ef finale de la respiration sont les mêmes que les valeurs correspondantes chez les larves non induites en diapause.

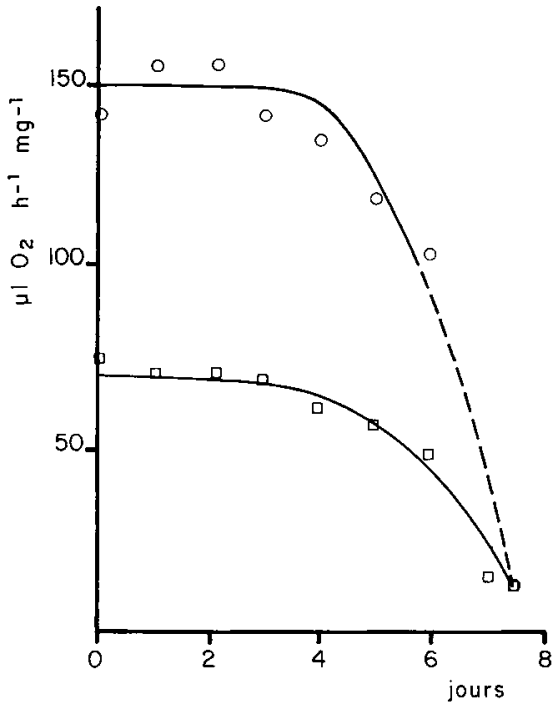

FIG. 6. - Activité respiratoire spécifique des mitochondries des larves $P C$ au cours du $5^{\mathrm{e}}$ age avant $\square$ et après o l'addition d'ADP. Substrat = succinate.

3b) Synthèse d'ATP.

- Chez les larves PL, en présence d' $\alpha$-glycérophosphate, la synthèse d'ATP décroît lentement de 73 à 60 nmoles. $\mathrm{mn}^{-1} \mathrm{mg}^{-1}$ de protéines, en 4 jours. Les variations sont plus marquées en présence de succinate : 230 nmoles les trois premiers jours et 115 nmoles le 4 e jour (fig. 7).

- Pour les larves PC (fig. 8), on retrouve des valeurs voisines : diminution de 84 à

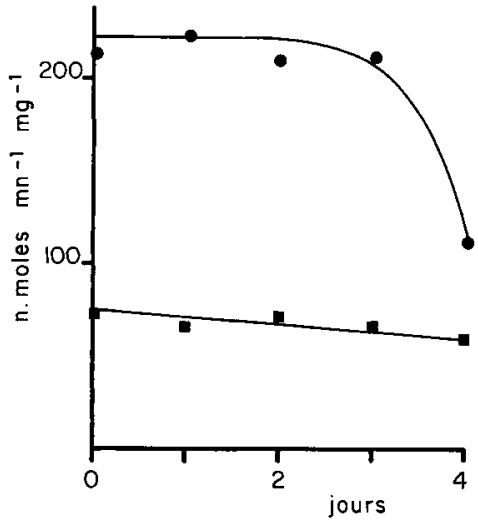

FIG. 7.

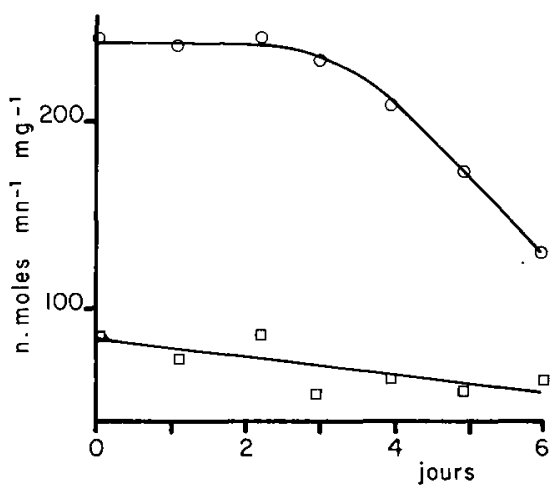

FIG. 8.

FIG. 7. - Synthèse d'ATP chez les larves PL. Substrat = succinate $\bullet$ ou $\alpha$-glycérophosphate $\bullet$.

FIG. 8. - Synthèse d'ATP chez les larves PC. Substrat = succinate o ou $\alpha$-glycérophosphate $\square$. 
62 nmoles en 6 jours après l' $\alpha$-glycérophosphate et de 240 à 130 nmoles avec le succinate. Avec la technique employée, l'absence de couplage au stade prénymphe nous prive de la dernière valeur traduisant la synthèse d'ATP.

3c) Phosphorylation oxydative et contrôle respiratoire.

Le tableau 2 montre l'évolution du $\mathrm{P} / \mathrm{O}$ et du contrôle respiratoire au cours du dernier âge larvaire, pour les deux substrats utilisés. On peut faire les remarques suivantes : le contrôle respiratoire est plus élevé avec le succinate que l' $\alpha$-glycérophosphate; le $\mathrm{P} / \mathrm{O}$ est à peu près constant avec le premier substrat ; par contre, avec l' $\alpha$-glycérophosphate, il diminue à partir du 3 e jour dans les deux catégories de larves.

\section{TABLEAU 2}

Evolution du $P / O$ et du contrôle respiratoire $(R C R)$ en présence de succinate ou d' $\alpha$-glycérophosphate au cours du 5 eage chez les larves soumises à une photopériode longue $P L$ ou à une photopériode courte $P C$

\begin{tabular}{|c|c|c|c|c|c|c|c|c|c|c|}
\hline Substrat & Jours & & 0 & 1 & 2 & 3 & 4 & 5 & 6 & 7 \\
\hline \multirow[b]{2}{*}{$\alpha-G P$} & $\mathrm{RCR}$ & $\begin{array}{l}\mathrm{PL} \\
\mathrm{PC}\end{array}$ & $\begin{array}{l}2,72 \\
3,40\end{array}$ & $\begin{array}{l}2,48 \\
2,90\end{array}$ & $\begin{array}{l}3,16 \\
2,76\end{array}$ & $\begin{array}{l}2,89 \\
3,03\end{array}$ & $\begin{array}{l}3,63 \\
2,80\end{array}$ & $\overline{3,01}$ & 3,47 & - \\
\hline & $P / O$ & $\begin{array}{l}\mathrm{PL} \\
\mathrm{PC}\end{array}$ & $\begin{array}{l}1,39 \\
1,33\end{array}$ & $\begin{array}{l}1,20 \\
1,10\end{array}$ & $\begin{array}{l}1,29 \\
1,11\end{array}$ & $\begin{array}{l}1,20 \\
0,88\end{array}$ & $\begin{array}{l}0,96 \\
1,05\end{array}$ & $\overline{0,9}$ & & - \\
\hline \multirow{2}{*}{ Succinate } & $\mathrm{RCR}$ & $\begin{array}{l}\text { PL } \\
\text { PC }\end{array}$ & $\begin{array}{l}3,17 \\
3,57\end{array}$ & $\begin{array}{l}3,46 \\
3,26\end{array}$ & $\begin{array}{l}3,84 \\
3,78\end{array}$ & $\begin{array}{l}3,70 \\
4,48\end{array}$ & $\begin{array}{l}3,98 \\
4,35\end{array}$ & $\overline{4.56}$ & 3,16 & - \\
\hline & $\mathbf{P} / \mathrm{O}$ & $\begin{array}{l}\text { PL } \\
\text { PC }\end{array}$ & $\begin{array}{l}1,22 \\
1,38\end{array}$ & $\begin{array}{r}1,30 \\
1,28\end{array}$ & $\begin{array}{l}1,30 \\
\mathbf{1}, 38\end{array}$ & $\begin{array}{l}1,29 \\
1,25\end{array}$ & $\begin{array}{l}1,34 \\
1,38\end{array}$ & $\overline{1,37}$ & 1,13 & - \\
\hline
\end{tabular}

\section{Discussion.}

1. La comparaison de la respiration des mitochondries extraites à celle de l'homogénat sous la forme RM/RH p. 100 permet de connaître le rendement de l'extraction. Pendant les 4 premiers jours du 5e âge chez les larves PL et pendant les 6 premiers chez les larves PC, les valeurs de ce rapport sont comprises entre 54 et $60 \mathrm{p}$. 100. Chez les prénymphes, cette valeur est de 12 p. 100. A ce moment du développement, les mitochondries sont devenues plus sensibles aux différentes étapes de l'extraction, centrifugation en particulier. La figure 1 montre que cette étape entraîne une perte de 50 p. 100 de l'activité. De plus, l'ADP ne stimule pas la respiration de ces mitochondries. Les figures 5 et 6 montrent qu'en présence de succinate, la respiration à l'état 3 est 2 à 2,2 fois supérieure à la respiration des mitochondries avant addition d'ADP. Si l'on tient compte à la fois de la perte d'activité et de la non stimulation de la respiration par l'ADP, on retrouve pour les prénymphes une valeur du rapport RM/RH de 53 p. 100. Dans ces conditions, on peut estimer que la fraction des mitochondries isolées par ce procédé est de l'ordre de 55 p. 100 pendant tout le 5 e stade. La méthode d'extraction des mitochondries par centrifugation différentielle donne ce résultat chez d'autres insectes (Keeley, 1973 ; Guillet, 1979). 
2. Chez Pieris, l'élevage en jours courts qui induit la diapause, se traduit par un allongement du dernier stade larvaire (Claret, 1968) ; la période de prise de nourriture est augmentée, les larves sont plus lourdes, l'intensité respiratoire de l'animal est plus forte, la quantité de protéines mitochondriales y est plus élevée. Lorsque ces paramètres sont exprimés par unité de poids vif, ces différences entre larves PL et larves PC disparaissent. Si l'on calcule les temps en pourcentage de la durée du $5^{\mathrm{e}}$ síade, on constate que les activités respiratoire et ATP-synthétique sont tout à fait comparables à un même moment de l'âge. Il existe donc simplement une différence de taille et de vitesse de développement qui se répercute sur divers systèmes physiologiques ou métaboliques, mais pas de différence fondamentale sur la nature des phénomènes.

3. Nos résultats montrent clairement qu'il existe deux phases successives dont la limite se situe entre 50 et 60 p. 100 de la durée du $5^{\mathrm{e}}$ stade larvaire.

Pendant la première phase, la croissance pondérale est rapide, la quantité de protéines totales augmente ainsi que la quantité de protéines mitochondriales estimée à partir du culot $8700 \mathrm{~g}$ : il faut souligner que 85 à $90 \mathrm{p} .100$ des protéines mitochondriales sont synthétisées sur les ribosomes cytoplasmiques (Bartelink et De Kort, 1973 ; Chaudhary et Malhotra, 1974). L'activité mitochondriale varie très peu.

Dans la seconde phase, les phénomènes de croissance s'amortissent, puis cessent. On assisie à un arrêt de l'alimentation. Les larves vident leur tube digestif. Le poids vif diminue ainsi que la respiration des larves, la quantité de protéines mitochondriales ef l'activité spécifique des mitochondries. Nous avons vérifié que cette dernière ne résulte pas d'une perte de cytochrome C qui n'apparaît à aucun moment comme facteur limitant.

- Dans l'âge larvaire, il est classique de reconnaître deux périodes successives. Ce sont les périodes d'alimentation obligatoire ef facultative (Legay, 1955 ; Fourche, 1967b). Une larve mise au jeûne dans la période d'alimentation obligatoire est incapable d'effectuer la mue suivante contrairement à ce qui se passe si la privation de nourriture intervient en période d'alimentation facultative. Le changement d'activité que nous observons se situe au moment où toutes les larves privées de nourriture sont capables d'effectuer, après une certaine atttente, la mue larvo-nymphale.

- Au niveau hormonal, au début de la première période, le taux d'ecdystéroïdes dans l'hémolymphe est très faible (Calvez, 1976 ; Lafont, 1976), le taux d'hormone juvénile est élevé (Varjas et al., 1976 ; Mauchamp et al., 1979). Cette dernière est connue pour agir sur les propriétés membranaires (Bauman, 1968) y compris au niveau mitochondrial (Firstenberg et Silhacek, 1973). Pendant cette période, la respiration spécifique des mitochondries est plus élevée en présence de succinate qu'en présence d' $\alpha$-glycérophosphate. Ensuite, le taux d'hormone juvénile devient très bas (Varjas et al., 1976 ; Mauchamp et al., 1979) et la différence de perméabilité des mitochondries vis-à-vis des deux substrats s'estompe pour devenir inexistante dans les mitochondries de prénymphes. Cette situation est l'inverse de celle rencontrée au cours de la maturation des sarcosomes (Sacktor, 1974).

La diminution d'activité des mitochondries se situe au moment du pic d'ecdystéroïdes dans l'hémolymphe (Calvez, 1976 ; Lafont, 1976), en présence de succinate et juste après le pic en présence d' $\alpha$-glycérophosphate. II faut remarquer que chez les larves PC où la diminution de l'activité est plus tardive, le pic d'ecdysone est aussi décalé. Chez Drosophila (Rezvoy, 1977), les figures de dégénérescence observées dans 
les culots mitochondriaux n'apparaissent qu'après la chute du taux d'ecdysone (Hodgetts et al., 1977). La présence de ces figures contribue à abaisser la respiration spécifique. Les modifications qui interviennent au niveau de la structure des mitochondries pourraient être apparentées aux processus d'histolyse déclenchés par un fort taux d'ecdysone (Dean, 1978). De plus, la quantité de mitochondries décroît à la fin de la vie larvaire comme le montrent nos résultats et ceux obtenus par d'autres auteurs (Walker, 1966 ; Brown et Chippendale, 1977), conséquence d'une forte activité autophagique (Locke et Collins, 1968).

4. L'hypothèse classiquement admise est que la demande énergétique régule le métabolisme oxydatif (Harvey, 1962) et selon Steele (1976) l'effet stimulant de certaines hormones sur la respiration n'est que le reflet des besoins en énergie provenant d'une activation des mécanismes de la physiologie du développement ou de la reproduction. Cette notion de modulation de la respiration en fonction de la demande énergétique existe très certainement. Cependant, nos résultats apportent des informations supplémentaires. In vitro, la respiration du culot mitochondrial $8700 \mathrm{~g}$ au cours du 5 e stade, varie toujours dans le même sens que la respiration de la larve. La respiration de ce culot fait intervenir à la fois la quantité de mitochondries et l'activité spécifique.

On peut dire que pendant la période d'augmentation pondérale de la larve, la quantité de protéines mitochondriales peut être considérée comme un élément de la croissance (Guillet, 1979). Pendant cette période, c'est en fin de compte l'augmentation de la quantité de mitochondries qui permet, à chaque instant, à la respiration de l'animal d'être ajustée à la demande énergétique. La croissance se caractérise donc par une augmentation synchrone des besoins et de la production en énergie.

En ce qui concerne l'activité spécifique, in vitro, les conditions étant standardisées, il n'est pas possible de dire que la production d'énergie par les mitochondries suit la demande énergétique. Dans des conditions d'élevage standard, au $5^{\mathrm{e}}$ stade larvaire, l'activité des mitochondries, à un instant donné, esł donc une caractéristique du développement.

En conclusion, le système mitochondrial s'intègre parfaitement dans un système programmé au sein duquel une cerfaine modulation reste possible et dans ces conditions, la respiration de l'animal, dont l'évolution est caractéristique d'une étape du développement, ne fait pas que suivre passivement la demande énergétique.

Reçu en juillef 1979

Accepté en janvier 1980.

\section{Références}

BARTELINK A., DE KORT C., 1973. Synthesis of mitochondrial protein in the flight muscle of the colorado beetle: Differentiation in vivo between extra- and intra-mitochondrial contributions to the amino-acid incorporation into mitochondrial protein. Biochem. J., 136, 795-802.

BAUMAN G., 1968. Zur Wirkung des Juvenilhormons : elektrophysiologische Messungen an der Zellmembran der Speicheldrüse von Galleria mellonella. J. Insect Physiol., 14, 1469-1476.

BOSQUET G., 1971. Evolution du métabolisme respiratoire ou cours du jeûne chez Bombyx mori. Th. Spécial., Lyon.

BROWN J. J., CHIPPENDALE G. M., 1977. Ultrastructure and respiration of the fat body of diapausing and non-diapausing larvae of the cornborer Diatrea grandiosella. J. Insect Physiol., 23, 1135-1142. 
CALVEZ B., 1976. Taux d'ecdysone circulante au dernier âge larvaire et induction de la diapause nymphale chez Pieris brassicae. C. R. Acad. Sci. Paris, 282D, 1367-1370.

CHAUDHARY K. D., MALHOTRA L. C., 1974. Effets de l'ecdysone sur l'incorporation in vivo des acides aminés dans les protéines des fractions mitochondriale ef post-mitochondriale du muscle thoracique de criquet, Schisfocerca gregaria. Rev. can. Biol., 33, 161-166.

CLARET J., 1968. Modifications physiologiques provoquées par la photopériode pendant la prédiapause chez la chenille de Pieris brassicae (L.). C. R. Acad. Sci. Paris, 266D, 1156-1159.

DEAN R. L., 1978. The induction of autophagy in isolated insect fat body by ecdysone. J. Insect Physiol., 24, 439-447.

ESTABROOK R. W., 1967. Mitochondrial respiratory control and the polarographic measurement of ADP : 0 ratio. In ESTABROOK R. W., PULHMAN M. E., Methods in enzymology, vol. 10, 41-57, Acad. Press, New-York.

FIRSTENBERG D. E., SILHACEK D. L., 1973. Juvenile hormone regulation of oxidative metabolism in isolated insect mitochondria. Experientia, 29, 1420-1422.

FOURCHE J., 1965. La respiration larvaire chez Drosophila melanogaster. Consommation d'oxygène au cours de la croissance. C. R. Acad. Sci. Paris, 261 D, 2965-2968.

FOURCHE J., 1967a. La respiration chez Drosophila melanogaster au cours de la métamorphose. Influence de la pupaison, de la mue nymphale ef de l'émergence. J. Insect Physiol., 13, 12691277.

FOURCHE J., 1967b. Le déterminisme des mues et des métamorphoses chez Drosophila melanogaster. Influence du jeône et de la fourniture d'ecdysone. Arch. Anat. microsc. Morph. exp., 56, 141-152.

FOURCHE J., 1969. Les relations entre la consommation d'oxygène et la morphogenèse au cours du développement de deux insectes holométaboles : Drosophila melanogaster (Diptère), Bombyx mori (Lépidoptère). Ann. Biol., 8, 334-367.

FOURCHE J., AMBROSIONI J.-C., 1969. Le métabolisme respiratoire au cours des métamorphoses. Respiration in vitro des ovaires de Bombyx mori. J. Insect Physiol., 15, 1981-1997.

FOURCHE J., FENOUILLET J.-P., BOSQUET G., 1972. Un respiromètre électronique automatique. Application à la mesure de la respiration au cours de la mue de Bombyx mori. Bull. Biol. Fr. ef Belg., 106, 143-158.

GUILLET C., 1976. Le métabolisme de diapause chez Pieris brassicae : les nucléotides adényliques. Ann. Biol., 15, 77-90.

GUILLET C., 1979. Relation entre poids vif, protéines mitochondriales, adénosine triphosphate au stade larvaire chez Pieris brassicoe (Lépidoptère) et Drosophila melanogaster (Diptère). C. $R$. Acad. Sci. Paris, 289D, 493-496.

HARVEY W. R., 1962. Metabolic aspects of insect diapause. Ann. Rev. Entomol., 7, 57-74.

HEROLD R. C., 1965. Development and ultrastructural changes of sarcosomes during Honey Bee flight muscle development. Devel. Biol., 12, 269-286.

HODGETTS R. B., SAGE B., O'CONNOR J. D., 1977. Ecdysone titers during post embryonic development of Drosophila melanogaster. Devel. Biol., 60, 310-317.

HOLMES E. A., KEELEY L. L., 1975. Metabolic inhibitors effects metamorphosis and flight muscle mitochondrial development in the moth Heliothis virescens. Insect Biochem., 5, 349-355.

KEELEY L. L., 1973. Characterization of insect fat body mitochondria isolated by a rapid procedure. Comp. Biochem. Physiol., 46B, 147-151.

KEELEY L. L., OLSON J. K., 1977. Toxic effects of mitochondrial DNA inhibitors on insect growth and development. J. Insect Physiol., 23, 303-307.

KUNKEL J. G., 1975. Cockroach molting. I. Temporal organization of events during the molting cycle of Blattela germanica. Biol. Bull. Woods Hole, 148, 259-273.

LAFONT R., 1976. Aspects biochimiques et endocrines de la métamorphose des lépidoptères. Bull. Soc. Zool. Fr., 101, 55-61, suppl, au no 5.

LARSEN W. J., 1970. Genesis of mitochondria in insect fat body. J. Cell Biol., 47, 373-383.

LEGAY J.-M., 1955. La prise de nourrifure chez le Ver à soie. Institut National de la Recherche Agronomique, Ed., Paris, 169 pp.

LOCKE M., COLLINS J. V., 1968. Protein uptake into multivesicular bodies and storage granules in the fat body of an insect. J. Cell Biol., 36, 453-483.

LOWRY O. H., ROSEBROUGH N. J., FARR A. L., RANDALL R. J., 1951. Protein measurement with Fo!in phenol reagent. J. biol. Chem., 193, 265-275. 
MAUCHAMP B., LAFONT R., JOURDAIN D., 1979. Mass fragmentographic analysis of juvenile hormone 1 levels during the last larval instar of Pieris brassicae. J. Insect Physiol., 25, 545-550.

REZVOY M., 1977. Etude du système mifochondrial pendant le développement larvaire de Drosophila melonogosfer. Th. Spécial., Lyon, 33 pp.

SACKTOR B., 1974. Biological oxidations and energetics in insect metamorphos. In Rockstein M., The physiology of Insecta Acad. Press, New-York and London.

SCHNEIDERMAN H. A., WILLIAMS C. M., 1953. The physiology of insect diapause. VII. The respiration metabolism of the Cecropia silkworm during diapause. Biol. Bull., 105, 382-389.

SLAMA K., 1968. Hormonal control of developmental and metabolic cycles in insects. Gen. comp. Endocrinol., 9, 492-493.

STEELE J. E., 1976. Hormonal conirol of metabolism in insects. Adv. Insect Physiol., 12, 239-323.

VARIAS L., PAGUIA P., DE WILDE J., 1976. Juvenile hormone titers in penultimate and last instar larvae of Pieris brassicae (Pieridae) and Barathra brassicae (Noctuidae) in relation to the effect of juvenoid application. Experientia, 32, 249-251.

WALKER A. C., BIRT L. M., 1969. Development of respiratory activity and oxydative phosphorylation in flight muscle mitochondria of the blowfly, Lucilia cuprina. J. Insect Physiol., 15, 305-317.

WALKER P. A., 1966. An electron microscope study of the fat body of the moth Philosamia during growth and metamorphosis. J. Insect Physiol., 12, 1009-1018.

WIGGLESWORTH V. B., 1949. Metabolism during flight and starvation in Drosophila. J. exp. Biol., 26, 150-153.

WIGGLESWORTH V. B., 1959. The control of growth and form. Cornell Univ. Press., Ithaca, N. Y., 55. 